\title{
Intermittent particle dynamics in marine coastal waters
}

\author{
P. R. Renosh ${ }^{1}$, F. G. Schmitt ${ }^{2}$, and H. Loisel $^{3}$ \\ ${ }^{1}$ University of Lille, UMR 8187, Laboratory of Oceanology and Geosciences, 28 Avenue Foch, 62930 Wimereux, France \\ ${ }^{2}$ CNRS, UMR 8187, Laboratory of Oceanology and Geosciences, 28 Avenue Foch, 62930 Wimereux, France \\ ${ }^{3}$ ULCO, UMR 8187, Laboratory of Oceanology and Geosciences, 32 Avenue Foch, 62930 Wimereux, France
}

Correspondence to: F. G. Schmitt (francois.schmitt@cnrs.fr)

Received: 23 May 2015 - Published in Nonlin. Processes Geophys. Discuss.: 18 July 2015

Revised: 19 September 2015 - Accepted: 7 October 2015 - Published: 22 October 2015

\begin{abstract}
Marine coastal processes are highly variable over different space scales and timescales. In this paper we analyse the intermittency properties of particle size distribution (PSD) recorded every second using a LISST instrument (Laser In-Situ Scattering and Transmissometry). The particle concentrations have been recorded over 32 size classes from 2.5 to $500 \mu \mathrm{m}$, at $1 \mathrm{~Hz}$ resolution. Such information is used to estimate at each time step the hyperbolic slope of the particle size distribution, and to consider its dynamics. Shannon entropy, as an indicator of the randomness, is estimated at each time step and its dynamics is analysed. Furthermore, particles are separated into four classes according to their size, and the intermittent properties of these classes are considered. The empirical mode decomposition (EMD) is used, associated with arbitrary-order Hilbert spectral analysis (AHSA), in order to retrieve scaling multifractal moment functions, for scales from $10 \mathrm{~s}$ to $8 \mathrm{~min}$. The intermittent properties of two other indicators of particle concentration are also considered in the same range of scales: the total volume concentration $C_{\text {vol-total }}$ and the particulate beam attenuation coefficient $c_{\mathrm{p}}(670)$. Both show quite similar intermittent dynamics and are characterised by the same exponents. Globally we find here negative Hurst exponents (meaning the small scales show larger fluctuation than large scales) for each time series considered, and nonlinear moment functions.
\end{abstract}

\section{Introduction}

Ocean data fields show a high variability over many different time and space scales. Such variability is often associated with turbulence, and multi-scaling properties of oceanic fields have been reported and studied in many previous studies: sea state (Kerman, 1993); phytoplankton concentration (Seuront et al., 1996a, b, 1999; Lovejoy et al., 2001a); rainfall and cloud radiance (Tessier et al., 1993; Lovejoy and Schertzer, 2006); and satellite images of ocean colour, chlorophyll $a$ and sea surface temperature (Lovejoy et al., 2001b; Nieves et al., 2007; Pottier et al., 2008; Turiel et al., 2009; de Montera et al., 2011; Renosh et al., 2015). Here we focus on coastal waters and consider particles transported by oceanic currents in this highly energetic medium (Svendsen, 1987; Schmitt et al., 2009). The solid phases in the environment have been described by hyperbolic particle size distribution (PSD) of clay aggregates in water (Amal et al., 1990), biological aggregate and marine snow (Jiang and Logan, 1991; Logan and Wilkinson, 1991), aerosol agglomerates (Wu and Friedlander, 1993) and flocs produced in the water, and wastewater discharge (Li and Ganczarczyk, 1989).

PSD has a major influence in biological, physical and chemical processes in the aquatic environment (Boss et al., 2001; Twardowski et al., 2001; Reynolds et al., 2010). For instance, PSD is strongly involved in the trophic interaction within the plankton community and in the chemical/geological aspects. The shape of the PSD is also used in computing the sinking rate of the sediment fluxes. The study carried out by Renosh et al. (2014) using the same in situ data set as the present study showed that the dynamics of the PSD is controlled by many oceanographic parameters like tidal currents, waves, and turbulence. The present study is a continuation of this work.

Most environmental and geophysical data sets are nonlinear and non-stationary at many different scales of time and space. Intermittency is a property that occurs in fully developed turbulence ranging between the large-scale injection and the small-scale dissipation (Frisch, 1995; Pope, 2000). 
The main objective of this study is to analyse the intermittency properties of particle size distribution (PSD). In this study we mainly focus on the dynamics of the PSD along with the velocity data. For that we decomposed the PSD into different size classes and also derived the Shannon entropy from the probability density function (PDF) of the PSD.

Empirical mode of decomposition (EMD) together with Hilbert spectral analysis (HSA) is a well-known timefrequency analysis method for non-stationary and nonlinear time series (Huang et al., 1998, 1999). Such analysis is done in two parts: the EMD is an algorithm to decompose a time series into a sum of mono-chromatic modes, and HSA extends for each mode into characteristic amplitude and frequency. Hence this method is a time-amplitude frequency analysis, which is recalled in Appendices A and B. This approach can be generalised to extract intermittency exponents (Huang et al., 2008, 2011). AHSA scaling exponent function $\xi(q)$ is related to the classical structure function scaling exponent $\zeta(q)$ by $\xi(q)=\zeta(q)+1$, where $q$ is the statistical moment. This is presented in Appendix C.

The first part of the paper presents the study area and in situ data, and contains the separation of different size classes and the hyperbolic shape of the PSD. Intermittency analysis using the EMD-AHSA method (presented in the appendices) is then provided in the next section.

\section{In situ data}

The measurements were conducted $50 \mathrm{~cm}$ from the bottom of coastal waters of the eastern English Channel at a fixed station $\left(5045.676^{\circ} \mathrm{N}, 0135.117^{\circ} \mathrm{E}\right)$ from 25 to 28 June 2012 (Fig. 1).

We consider here simultaneous measurements of velocity and particle concentrations. The in situ sampling of Laser InSitu Scattering and Transmissometry (LISST 100X type C) has been carried out at $1.0 \mathrm{~Hz}$. The main part of the instrument is a collimated laser diode and a specially constructed annular ring detector. The primary information collected by the LISST is the scattering of the laser at 32 angles, which are converted into size distribution using an inverting method. The size distribution is presented as volume concentration with units of micro-litres per litre $\left(\mu \mathrm{LL}^{-1}\right)$. The LISST measures the volume concentration $C_{\mathrm{vol}, i}$ of particles having diameters ranging from 2.5 to $500 \mu \mathrm{m}$ in 32 size classes in logarithmic scale (Agrawal and Pottsmith, 2000). Because of instability in the smallest and largest size classes, the data recorded in the inner and outer rings are excluded from further analysis (Traykovski et al., 1999; Jouon et al., 2008; Neukermans et al., 2012). These instabilities observed in the smaller size classes have also been related to effects of stray light (Reynolds et al., 2010). The LISST also records the beam attenuation $(c)$ at $670 \mathrm{~nm}( \pm 0.1 \mathrm{~nm})$ over a $5 \mathrm{~cm}$ path length with an acceptance angle of $0.0135^{\circ}$. The particulate attenuation coefficient $c_{\mathrm{p}}$ has been derived from $c$

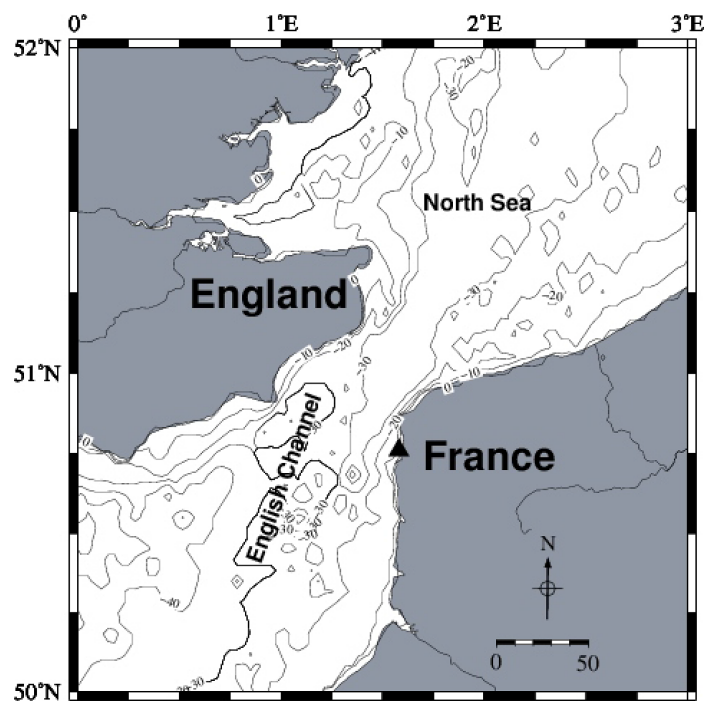

Figure 1. Location (black triangle) of the sampling station in the eastern English Channel together with the isobaths.

after calibration with MilliQ water before and after the field campaign, using the assumption that chromophoric dissolved organic matter (CDOM) does not absorb light at $670 \mathrm{~nm}$. $c_{\mathrm{p}}(670)$ is an important parameter which is directly linked to the suspended particulate matter (SPM) of the water body (Boss et al., 2009; Neukermans et al., 2012). Simultaneously, velocity time series are measured using a Nortek Vector ADV current meter fixed on the same platform along with the LISST at $0.5 \mathrm{~m}$ above the sea bottom. The ADV measured the north, east and up components of velocity with an accuracy of $\pm 0.5 \%$.

\subsection{Separation into size classes}

The volume concentration distributed of a particle size class can also be expressed as the concentration $C_{\mathrm{vol}}(\sigma)$ per unit volume per unit bin width (Jouon et al., 2008):

$$
C_{\mathrm{vol}}(\sigma)=\frac{C_{\mathrm{vol}, i}}{\sigma_{\max }(i)-\sigma_{\min }(i)},
$$

where $\sigma$ is the median diameter of the particle size class $i$, and $\sigma_{\max }(i)$ and $\sigma_{\min }(i)$ are respectively the maximum and minimum particle size of the class $i$. This resulting volumetric PSD is expressed in $\mu \mathrm{LL}^{-1} \mu \mathrm{m}^{-1}$. The total volume concentration of the PSD $\left(C_{\text {vol-total }}\right)$ has been derived at each time step:

$C_{\text {vol-total }}(t)=\sum_{i=6}^{31} C_{\mathrm{vol}}, i(t)$.

This quantity gives the total volume of the particles in $\mu \mathrm{LL}^{-1}$. For the present study we consider four different size classes, using the following classification: silt/clay $(\sigma<30 \mu \mathrm{m})$, fine $(30<\sigma<105 \mu \mathrm{m})$, coarse/micro 

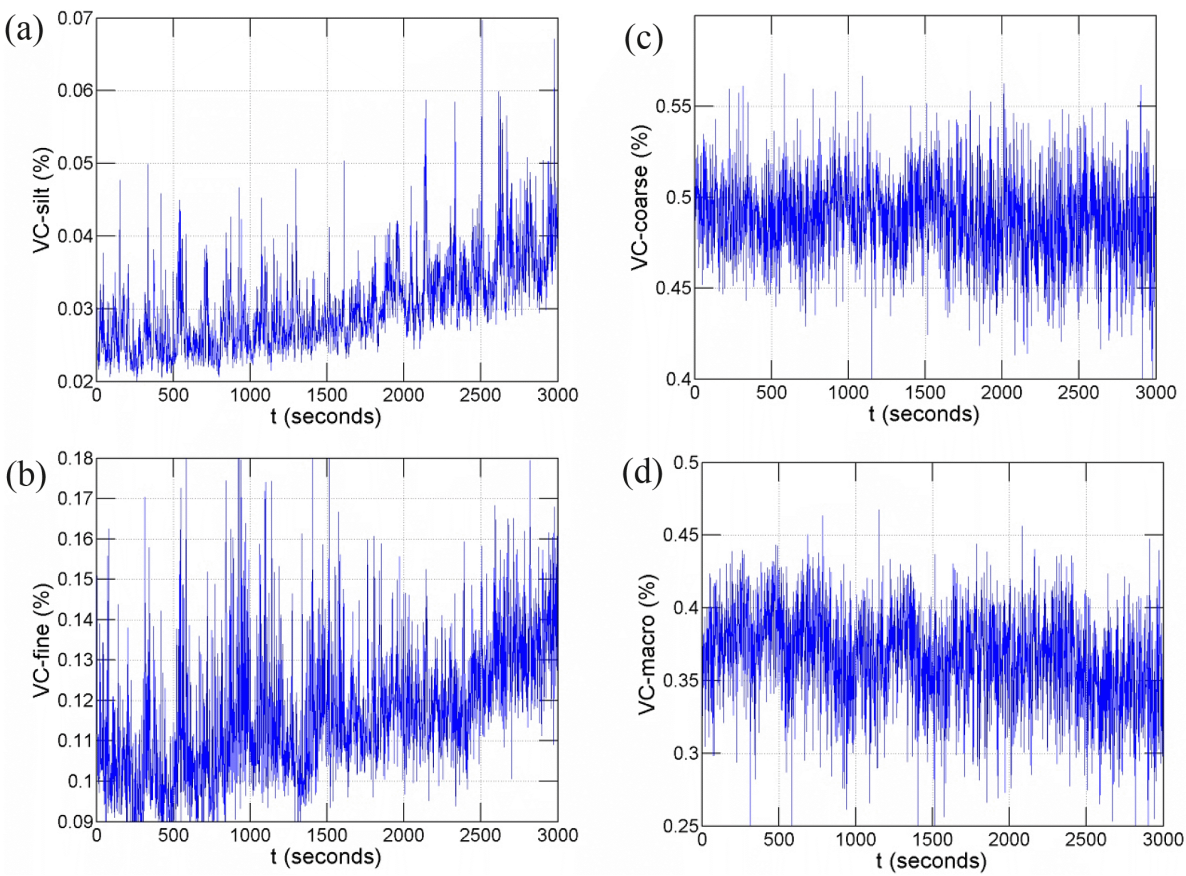

Figure 2. The first 3000 samples of the time series of volume concentrations of different size classes of PSD. (a) Silt/clay, (b) fine particles, (c) coarse/micro particles and (d) macro particles/flocs.

$(105<\sigma<300 \mu \mathrm{m})$ and macro flocs/particles $(\sigma>300 \mu \mathrm{m})$ (Lefebvre et al., 2012; Renosh et al., 2014). Figure 2 shows the time series of normalised volume concentrations (VC) of different size classes of PSD. All four size classes show large temporal fluctuations in their magnitude. Their statistical and dynamical properties are considered below.

\subsection{PSD slope $(\xi)$}

The particle size distribution in the ocean, which describes the particle concentration as a function of particle size/number, typically shows a rapid decrease in concentration with increasing size from a sub-micrometre range to hundreds of micrometres. This feature is common to all the suspended particles and also for plankton micro-organisms (Sheldon et al., 1972; McCave, 1983; Stramski and Kiefer, 1991; Jackson et al., 1997). The number of particles for a given size $\sigma$ is estimated by a normalisation by their volume (Jouon et al., 2008). We obtain the number density $n(\sigma)$, which is also the product of the probability density function of the size, $p(\sigma)$, times $N$, the total number of particles:

$n(\sigma)=N p(\sigma)=\frac{C_{\mathrm{vol}}(\sigma)}{\frac{4}{3} \pi(\sigma / 2)^{3}}$.

The PSD of this density number classically follows a powerlaw distribution for aquatic particles in suspension (Sheldon et al., 1972; Kitchen et al., 1982; Jonaszz, 1983; Boss et al., 2001; Twardowski et al., 2001; Loisel et al., 2006; Reynolds et al., 2010; Renosh et al., 2014): $n(\sigma) \sim K \sigma^{-\xi}$,

where $K$ is a constant and $\xi>0$ is the PSD hyperbolic slope. Since the LISST provides size class information at each time step, the power-law distribution can be fitted at each time step, providing the exponent as a time series $\xi(t)$. The $\xi$ value provides information on the relative concentration of small and large particles: the steeper the slope (the greater $\xi$ ), the more small particles relative to large particles are present in the water (and vice versa). A small portion of 3000 samples of $\xi$ is shown in Fig. 3a: large temporal fluctuations in its magnitude are visible. When considering all size classes in all the time steps, a hyperbolic PDF is also obtained, represented in Fig. 3b with a slope value of $\bar{\xi}=2.9 \pm 0.16$.

The study carried out by Renosh et al. (2014) considered the dynamics of the $\xi(t)$ in relation to different hydrodynamic quantities like waves, tidal currents and turbulence. It showed that turbulence has a major role in the re-suspension of the particles in the aquatic environment. It also showed that along-shore $(U)$ and cross-shore $(V)$ components of velocity have power spectra showing different scaling regimes in low-frequency and high-frequency regions (Fig. 4). At the low-frequency scale there is a typical Kolmogorov $-5 / 3$ slope and at high frequency a scaling regime with a 0.6 slope. For high frequencies there is a hump-like structure, which can be identified as the high energy associated with surf zone wave breaking (Schmitt et al., 2009).

The study of Renosh et al. (2014) showed that the lowfrequency variability of $\xi(t)$ and $c_{\mathrm{p}}(670)$ is controlled by 

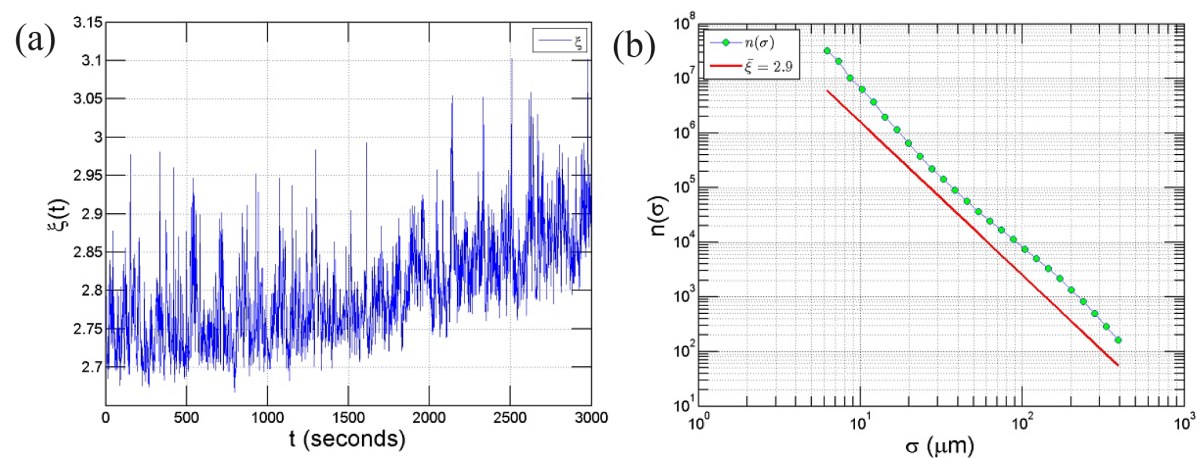

Figure 3. The first 3000 samples of the time series of PSD slope ( $\xi$ ) (a) and PSD slope of the entire data set with a power-law fit with a slope value of $\bar{\xi}=2.9 \pm 0.16(\mathbf{b})$.

turbulence and that the high-frequency part is related to dynamical processes impacted by the sea bottom. The present study is a continuation of Renosh et al. (2014); it considers the high-frequency scaling regimes and studies the intermittency of particle concentration in this range of scales.

\section{Intermittent dynamics}

\subsection{Velocity intermittency}

We first consider here the scaling and intermittency properties of the velocity. Figure 4a shows the Fourier and Hilbert (HSA) estimations of the $U$ and $V$ components of the velocity. Scaling ranges are found from 20 to $500 \mathrm{~s}$ with a slope of about -0.6 . In this range of scales the AHSA method has been applied to characterise intermittency in a multi-fractal framework (see Appendix $\mathrm{C}$ for the AHSA method). First a negative Hurst exponent is found: $H_{U}=-0.30 \pm 0.02$ and $H_{V}=-0.20 \pm 0.02$. Such a negative sign for $H$ values indicates that small scales show larger fluctuations than the larger scales in a scaling way (Lovejoy and Schertzer, 2012). Both curves become quite different for larger moments: the $U$ curve is more nonlinear, associated with larger intermittency (Fig. 4b).

\subsection{Dynamics of the entropy of particle size}

The LISST system records at each time step a discretised PDF of the particle size. Hence it is possible to estimate at all time steps the entropy of the particle size distribution as

$S(t)=-\sum_{i=6}^{31} P_{i}(t) \log P_{i}(t)$,

where $P_{i}(t)=n\left(\sigma_{i}\right)(t) / N(t)$. The Shannon entropy $S(t)$ is estimated at each time step with values centered around $\bar{S}=1.59 \pm 0.03$. Figure 5a shows a sample of $S(t)$ and Fig. 5b shows its PDF, which is centered around $\bar{S}$ with values ranging mainly between 1.5 and 1.7. As a stochastic process, in order to consider the dynamics of $S(t)$, we plot in
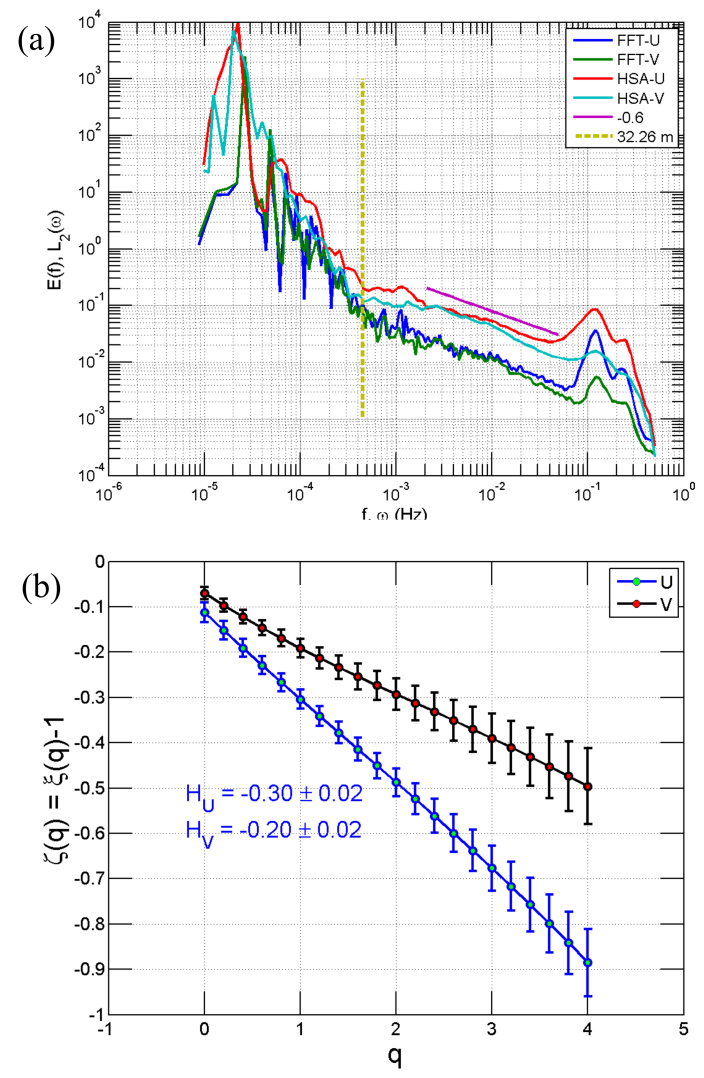

Figure 4. Turbulent power spectra of $U$ and $V$ components of velocity fields showing different scaling regimes as calculated by both FFT and HSA (a). The scaling exponents estimated using the HSA method (b). The vertical line in (a) shows the memory time of 36.26 min found in Eq. (6).

Fig. 5c the autocorrelation of $S(t)$. A memory time of the entropy series can be estimated as

$T=\int_{0}^{T_{0}} C_{\mathrm{s}}(t) \mathrm{d} t$, 

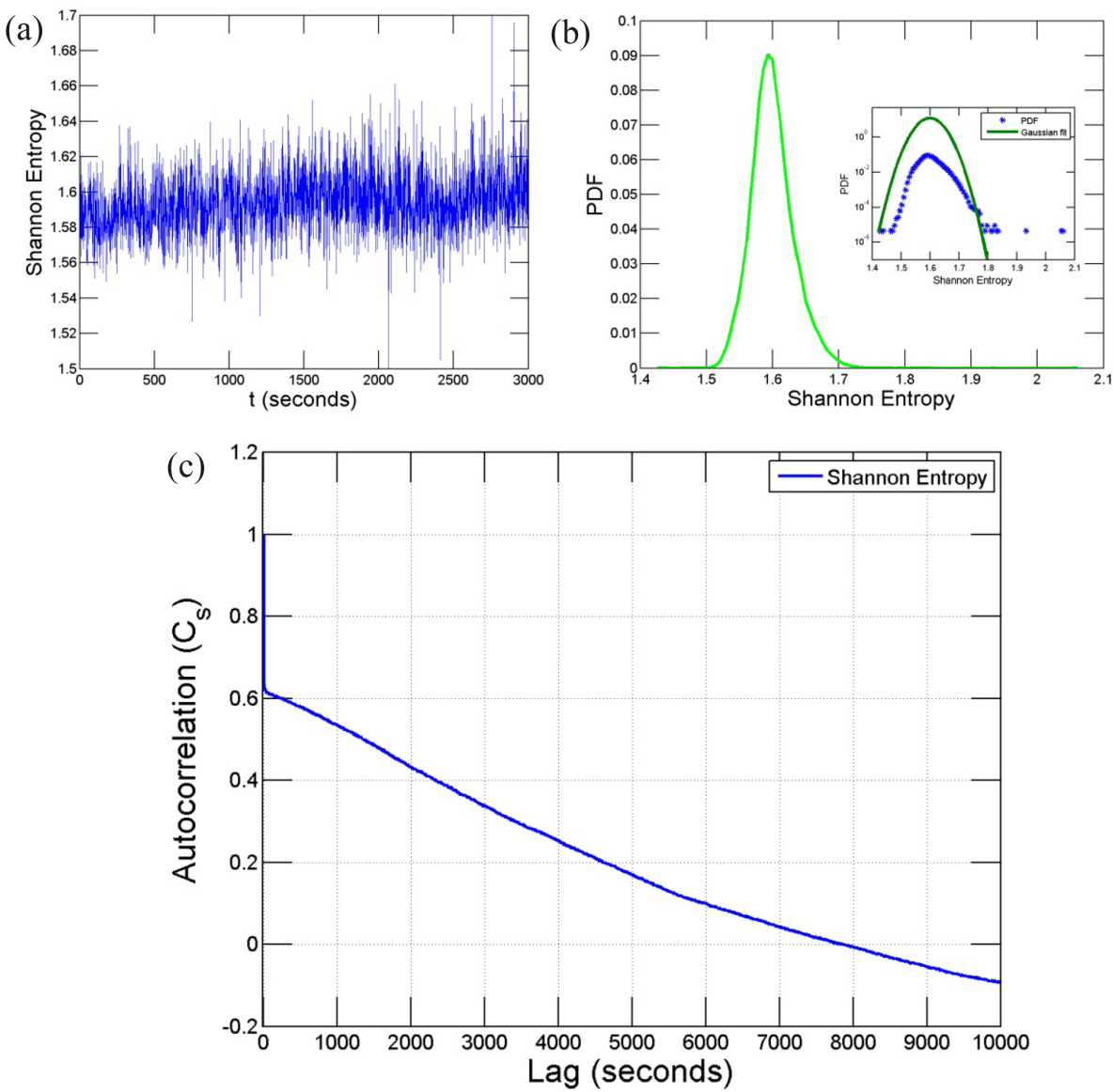

Figure 5. The first 3000 samples of the time series of Shannon entropy in (a), PDF of Shannon entropy along with a Gaussian fit in semi-log plot (inset) in (b) and the autocorrelation of Shannon entropy in (c).

where $C_{\mathrm{s}}$ is the autocorrelation of the entropy $S$ and $T_{0}$ is the first time for which $C_{\mathrm{s}}(t)=0$; we find here $T_{0}=7826 \mathrm{~s}$ and we compute $T=2176 \mathrm{~s}=36.26 \mathrm{~min}$. This characteristic timescale could be related to the transition scale (Fig. 4a) between two scaling regimes of low-frequency injection scale and high-frequency wave-breaking scale.

The entropy of particle sizes characterises the "disorder" of the size distribution, its information content. We showed here that the dynamics of such a quantity can be considered by using LISST data. A very interesting feature of LISST measurements is hence to be able to characterise nonlinear classical indicators such as the Shannon entropy in a dynamical way.

\subsection{Intermittent dynamics of different size classes}

As explained above, the PSD is decomposed into four different size classes of particles (silt/clay, fine particles, coarse/micro particles and macro particles/flocs). The power spectra of these four size classes have been derived using Fourier as well as Hilbert transforms (Fig. 6) for understanding the turbulent characteristics. Similar spectra are found from Fourier and Hilbert transforms, and there is a good power-law behaviour observed in the high-frequency region $(0.09-0.002 \mathrm{~Hz})$.

This scale range has been taken for the extraction of the scaling exponents. The scaling exponent function $\xi(q)$ has been extracted for all size classes using arbitraryorder Hilbert spectral analysis (Appendix C). The exponent $\zeta(q)=\xi(q)-1$ is computed. Nonlinear functions are visible for each size class (Fig. 7). The Hurst number $H=\zeta(1)=\xi(1)-1$ is estimated for each class: we find that $H=-0.17 \pm 0.01,-0.19 \pm 0.01,-0.38 \pm 0.02$, and $-0.26 \pm 0.02$ for increasing size classes. The high $H$ values are observed in the larger size classes and low $H$ values are observed in the lower size classes. This parameter determines the rate at which mean fluctuations grow $(H>0)$ or decrease $(H<0)$ with the scale. We found negative $H$ values in the present study. Negative $H$ values have not been found in many studies. Recently in Lovejoy and Schertzer $(2012,2013)$ it was argued that Haar wavelet analysis can be used to extract the $H$ values with any sign for the exponent $(-1<H<1)$. Such a sign indicates that small scales show larger fluctuation than large scales. If $\zeta(q)$ is linear, 

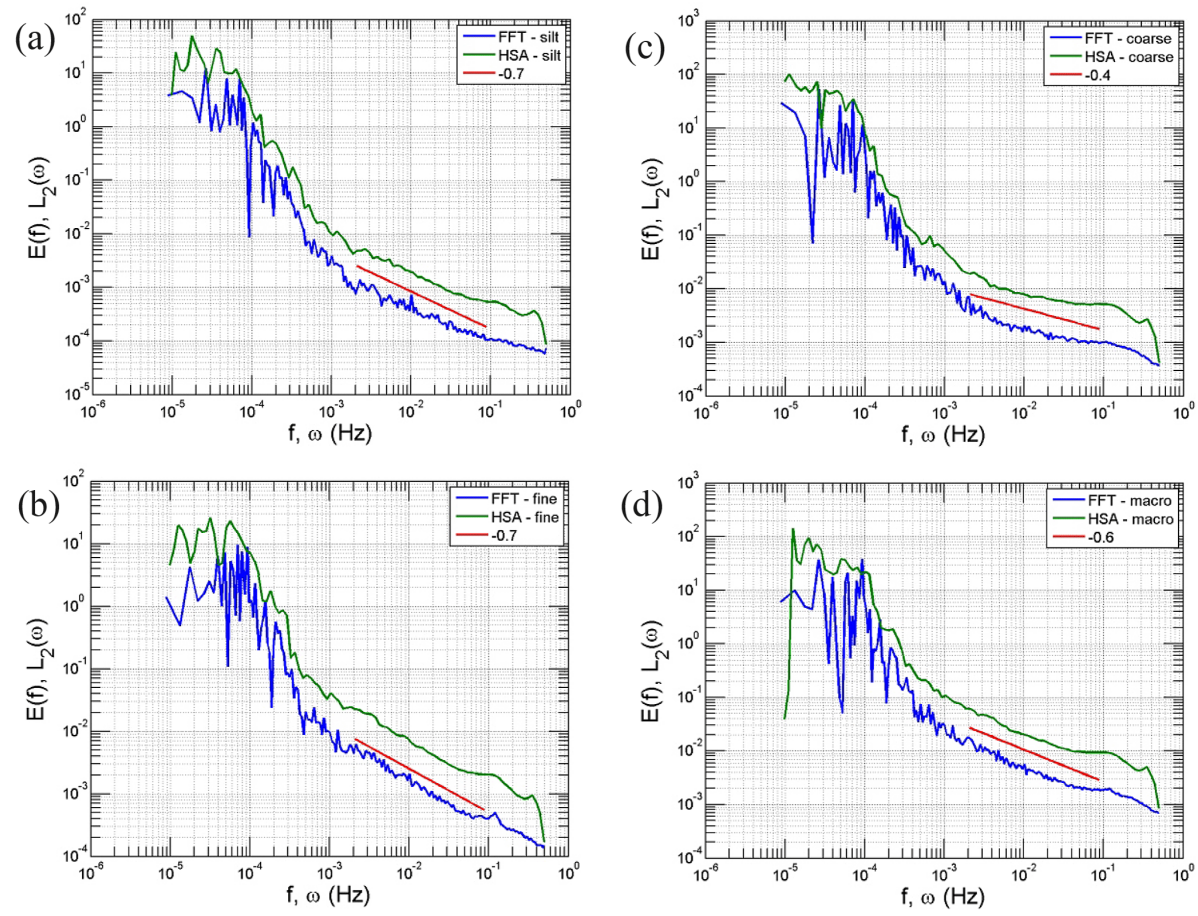

Figure 6. Power spectra for different size classes of PSD estimated for Fourier and Hilbert transforms. Silt/clay (a), fine (b), coarse/micro (c) and macro particles/flocs (d). The red lines shows the scaling range and the slope of the best fit in this range.

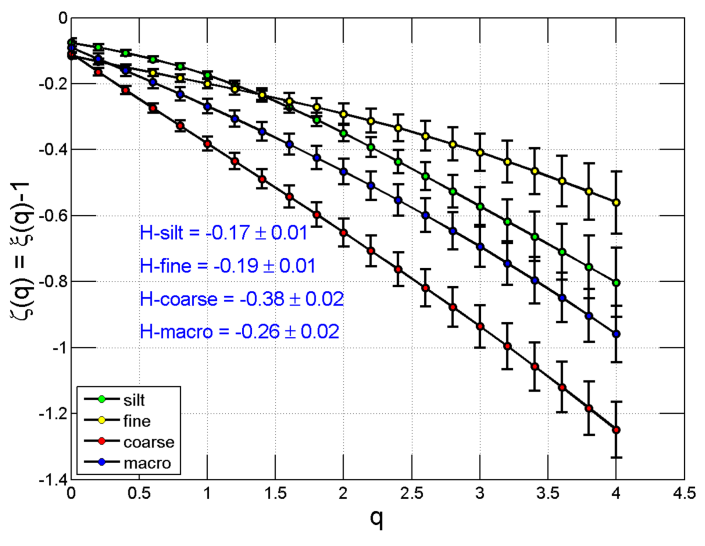

Figure 7. Scaling exponents $\zeta(q)$ estimated for different particle sizes using the HSA method.

the statistical behaviour is mono-scaling; if $\zeta(q)$ is nonlinear and concave/convex, the behaviour is defined as multiscaling, corresponding to a multifractal process. The concavity of this function is a characteristic of the intermittency: the more concave the curve is, the more intermittent the process is (Frisch, 1995; Schertzer et al., 1997; Vulpiani and Livi, 2003; Lovejoy and Schertzer, 2012). The slight curvature which is found here for all size classes (Fig. 7) is hence a signature of intermittency in the particle dynamics.

\subsection{Intermittent concentration dynamics}

We perform here an analysis of intermittency of concentration dynamics considering two indicators of this particle concentration: $c_{\mathrm{p}}(670)$ and total volume concentration $\left(C_{\text {vol-total }}\right)$. At first order, $c_{\mathrm{p}}(670)$ is driven by the suspended particulate matter (SPM). We observe here a large variability in the $c_{\mathrm{p}}(670)$ data (Fig. 8a). The total volume concentration of the PSD has been derived for each time step using Eq. (2). The derived $C_{\text {vol-total }}$ shows large fluctuation in its magnitude (Fig. 8b). The turbulent power spectra derived for these series show two scaling regimes similar to the size classes (Fig. 8c and d). A good scaling between 0.002 and $0.09 \mathrm{~Hz}$ with a $\beta$ value of 0.8 for $c_{\mathrm{p}}(670)$ and of 0.9 for $C_{\text {vol-total }}$ for the power spectra $E(f)$ of the form $E(f) \sim f^{-\beta}$ is observed (Fig. 8c and d). Hence the region between 0.002 and $0.09 \mathrm{~Hz}(10 \mathrm{~s}$ to $8 \mathrm{~min})$ has been identified for the multi-scaling analysis. The structure function scaling moment function derived for this series shows a nonlinearity and concavity in its shape (Fig. 8e). The $H$ value derived for the $C_{\text {vol-total }}$ is slightly negative: $H=-0.08 \pm 0.01$. The scaling moment function of the $c_{\mathrm{p}}(670)$ showed a nonlinearity in its behaviour showing its intermittent characteristics (Fig. 8e). We find here $H=-0.06 \pm 0.01$, which is quite similar to $C_{\text {vol-total }}$. Globally, for power spectra as well as for their intermittency properties, both proxies of SPM show similar scaling properties. These two different indicators of 

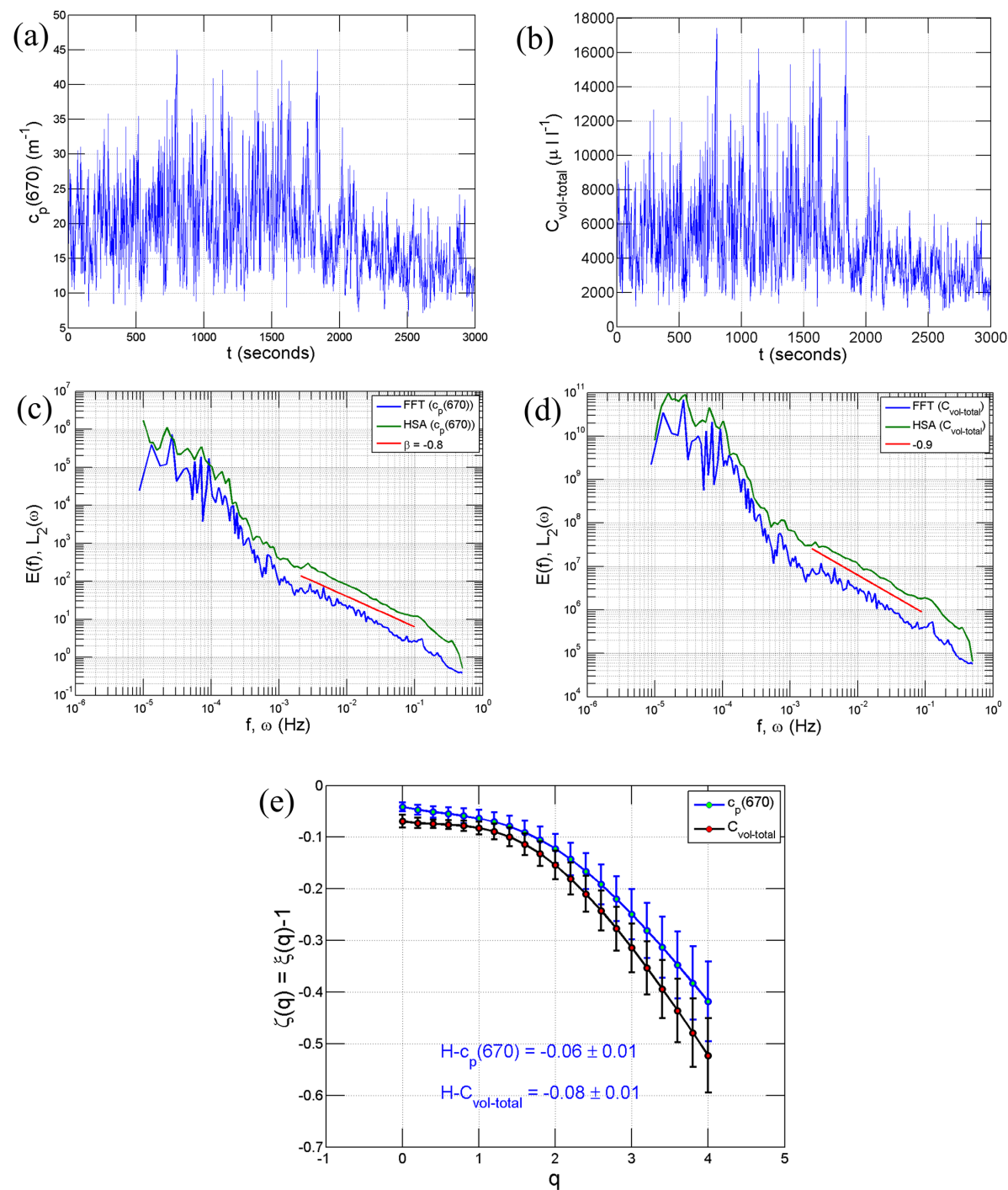

Figure 8. The first 3000 samples of the time series of $c_{\mathrm{p}}(670)$ in (a), the first 3000 samples of the time series of $C_{\mathrm{vol}-\text { total }}$ in (b), the turbulent power spectrum of $c_{\mathrm{p}}(670)$ and the turbulent power spectrum of $C_{\mathrm{vol} \text {-total }}$ showing different scaling regimes (the scaling regime indicated as red is used for the scaling exponent computation) in (c, d) and the scaling moment function of $c_{\mathrm{p}}(670)$ and $C_{\mathrm{vol}-\text { total }}$ in (e).

particle concentrations show quite similar dynamics and statistically intermittent properties.

For comparison purposes, the Haar wavelet structure function method, which can also be used for negative $H$ values (Lovejoy and Schertzer, 2012, 2013), has also been applied to the time series. The first-order Haar structure function has been selected for the Hurst number estimation. The same scaling region as for AHSA has been chosen for this analysis. Negative Hurst exponents for all parameters have been found, with values similar to those from the AHSA method. In some cases, there are some slight differences (Table 1).

An interesting point that can be noticed for these time series is that none of the scaling moment functions extracted through the AHSA method for various parameters showed $\zeta(0)=0$. This is due to the fact that a large number of
$\Delta X$ values are equal to zero, where $X$ is the time series: $\zeta(0)=0$ only if there are no zeros in the time series. When $H<0$, such a situation is more likely than when $H>0$, because the series is noisier.

\section{Conclusions}

This work analysed the intermittency and scaling properties of particles using the AHSA method. The intermittent transport of particles in complex flows, like in coastal waters, is very important for the study of partition dynamics, erosion processes, ecosystem modelling, sediment transport and turbidity dynamics. Suspended particle dynamics in turbulent flows are complex: some studies showed preferential concen- 
Table 1. The Hurst exponent values derived through AHSA and the Haar wavelet method for various parameters.

\begin{tabular}{lcc}
\hline Parameter & $H$ (AHSA) & $H$ (Haar wavelet) \\
\hline$U$ & $-0.30 \pm 0.02$ & $-0.25 \pm 0.03$ \\
$V$ & $-0.20 \pm 0.02$ & $-0.20 \pm 0.01$ \\
Silt & $-0.17 \pm 0.01$ & $-0.09 \pm 0.01$ \\
Fine & $-0.19 \pm 0.01$ & $-0.10 \pm 0.01$ \\
Coarse & $-0.38 \pm 0.02$ & $-0.24 \pm 0.04$ \\
Macro & $-0.26 \pm 0.02$ & $-0.18 \pm 0.02$ \\
$c_{\mathrm{p}}(670)$ & $-0.06 \pm 0.01$ & $-0.02 \pm 0.01$ \\
$C_{\text {vol-total }}$ & $-0.08 \pm 0.01$ & $-0.03 \pm 0.01$ \\
\hline
\end{tabular}

tration (Eaton and Fessler, 1994; Squires and Eaton, 1991) and some other studies showed multifractal repartition according to the Stokes number (Bec, 2005; Yoshimoto and Goto, 2007). We thus also expect here, in the natural environment, to find intermittent particle dynamics.

This work has analysed the intermittency and scaling properties of the PSD using different aspects. Time series of normalised volume concentrations of different size classes of PSD and Shannon entropy have been derived from the number density of PSD. Here we showed the intermittency of particles for different size classes. The $c_{\mathrm{p}}(670)$, a proxy of the suspended sediment concentration, and the total volume concentration $\left(C_{\text {vol-total }}\right)$, showed intermittent and multiscaling properties in their dynamics.
Turbulent scaling of these parameters has been derived through both Fourier power spectra and spectra derived through HSA. The scaling moment function derived for $C_{\text {vol-total }}$ and $c_{\mathrm{p}}(670)$ show similar nonlinear curve stressing the intermittency in their dynamics. The scaling moment functions derived for each size class of the particle are also nonlinear. The curvature of the spectrum for various size class shows the intermittency of the particle dynamics in different sizes.

We may note also that the Hurst exponents derived for the velocity components and the particle concentrations are negative. This negative sign indicates that small scales show larger fluctuations than large scales. We have here no theoretical interpretation to propose to explain these values, which could be related to the particular statistical characteristics of a bottom boundary-layer flow.

This multiscaling analysis has been tested only in the bottom of the highly dynamic coastal waters of the eastern English channel. Such an analysis is an illustration of the potential provided by LISST data, with many particle size classes recorded at each time step. It may be applied to other time series in the open ocean, coastal waters and also freshwater situations, in order to provide comparison and help to look for universal properties. 


\section{Appendix A: Empirical mode of decomposition (EMD)}

Hilbert spectral analysis (HSA) and empirical mode of decomposition (EMD) were introduced by Norden Huang and collaborators at the end of the 1990s (Huang et al., 1998) to locally extract amplitude and frequency information in a time series. They were mainly introduced for nonlinear and non-stationary time series. The first step of this approach is EMD. The objective of the EMD method is to decompose a signal into a series of modes. Each component is defined as an intrinsic mode function (IMF) satisfying the following conditions: (1) in the whole data set, the number of extrema and the number of zero crossings must either equal or differ at most by one. (2) The mean value of the envelope defined using the local maxima and the envelope defined using the local minima are zero (Huang et al., 1998; Huang and Wu, 2008). An iterative algorithm was proposed to extract successive IMF from time series. We do not reproduce all the details of this algorithm here, and refer to the original publications (Huang et al., 1998, 1999).

The decomposition process stops when the residue, $r_{n}$, becomes a monotonic function or a function with only one extremum from which no more IMF can be extracted. At the end of the decomposition, the original time series $x(t)$ is decomposed into a sum of $n$ modes and a residue:

$x(t)=\sum_{j=1}^{n} c_{j}(t)+r_{n}(t)$,

where $c_{j}(t)$ are IMFs and $r_{n}(t)$ is the residue. In this decomposition, each mode has a decreasing characteristic frequency. If $N$ is the number of points of the original series, we have $n \approx \log _{2}(N)$; hence, in general, $10 \leq n<20$ (Flandrin and Goncalves, 2004; Huang et al., 2008).

\section{Appendix B: Hilbert spectral analysis (HSA)}

Hilbert spectral analysis (HSA) is the second step of the analysis, which is applied to each mode $c_{j}(t)$ extracted for the time series $x(t)$ using the procedure discussed in Appendix A. For any function $x(t)$, its Hilbert transform $y(t)$ is written as

$y(t)=H\{x\}(t)=\frac{1}{\pi} \int_{-\infty}^{+\infty} \frac{x(\tau)}{t-\tau} \mathrm{d} \tau$.

The analytic function $z(t)$ is estimated from $x(t)$ using the Hilbert transform $y(t)$ :

$z(t)=x(t)+i y(t)=x(t)+i H\{x\}(t)$,

where $i=\sqrt{-1}$. The analytical function is estimated for each mode and at each time step. For each mode and each time step, a local amplitude $A$ and phase function $\theta$ can be estimated:

$A(t)=\left(x^{2}+y^{2}\right)^{1 / 2}$,

$\theta(t)=\tan ^{-1}(y / x)$.

The local frequency is estimated from the phase function:

$\omega=\frac{\mathrm{d} \theta}{\mathrm{d} t}$.

The HSA represents a time-amplitude frequency analysis. This helps to estimate a joint PDF $p(\omega, A)$ of frequency and amplitude. From this, a marginal spectrum is estimated:

$h(\omega)=\int_{0}^{\infty} p(\omega, A) A^{2} \mathrm{~d} A$.

This $h(\omega)$ spectral analysis is done through a Hilbert transform and can be compared to the Fourier spectrum $E(f)$ obtained through the classical Fourier analysis (Huang et al., 2008).

\section{Appendix C: Arbitrary-order Hilbert spectral analysis (AHSA)}

The equation obtained in the previous section giving $h(\omega)$ is a second-order statistical moment; it can be generalised into an arbitrary-order moment (Huang et al., 2008, 2011), by taking a moment of order $q$ :

$L_{q}(\omega)=\int_{0}^{\infty} p(\omega, A) A^{q} \mathrm{~d} A$,

where $q \geq 0$. In case of scale invariance we can write

$L_{q}(\omega) \approx \omega^{-\xi(q)}$,

where $\xi(q)$ is the corresponding scaling exponent, which is related to the classical structure function by $\xi(q)=1+\zeta(q)$ (Huang et al., 2008), for example, for a fractional Brownian motion $\xi(q)=1+q H$. Here we are interested in the Hurst exponent given by $H=\zeta(1)=\xi(1)-1$. $H$ can be positive or negative, and it characterises the degree of stationarity of the scaling process. The nonlinearity of $\zeta(q)$ is related to the intermittency of the time series: the more nonlinear the scaling exponent $\zeta(q)$, the more intermittent the series (Schmitt and Huang, 2015). 
Acknowledgements. The Centre National d'Etudes Spatiales (CNES) and the Centre National de la Recherche Scientifique (CNRS) are acknowledged for the funding of P. R. Renosh's $\mathrm{PhD}$ thesis. This study is performed in the framework of the COULCOT-2 project, funded by the CNES/TOSCA programme.

Edited by: A. Baas

Reviewed by: two anonymous referees

\section{References}

Agrawal, Y. and Pottsmith, H.: Instruments for particle size and settling velocity observations in sediment transport, Mar. Geol., 168, 89-114, 2000.

Amal, R., Raper, J. A., and Waite, T. D.: Fractal structure of hematite aggregates, J. Colloid Interf. Sci., 140, 158-168, 1990.

Bec, J.: Multifractal concentrations of inertial particles in smooth random flows, J. Fluid Mech., 528, 255-277, 2005.

Boss, E., Pegau, W. S., Gardner, W. D., Zaneveld, J. R. V., Barnard, A. H., Twardowski, M. S., Chang, G., and Dickey, T.: Spectral particulate attenuation and particle size distribution in the bottom boundary layer of a continental shelf, J. Geophys. Res.-Oceans, 106, 9509-9516, 2001.

Boss, E., Slade, W., and Hill, P.: Effect of particulate aggregation in aquatic environments on the beam attenuation and its utility as a proxy for particulate mass, Opt. Express, 17, 9408-9420, 2009.

de Montera, L., Jouini, M., Verrier, S., Thiria, S., and Crepon, M.: Multifractal analysis of oceanic chlorophyll maps remotely sensed from space, Ocean Sci., 7, 219-229, doi:10.5194/os-7219-2011, 2011.

Eaton, J. K. and Fessler, J.: Preferential concentration of particles by turbulence, Int. J. Multiphas. Flow, 20, 169-209, 1994.

Flandrin, P. and Goncalves, P.: Empirical mode decompositions as data driven wavelet-like expansions, Int. J. Wavelets Multi., 2, 477-496, 2004.

Frisch, U.: Turbulence: The Legacy of AN Kolmogorov, Cambridge University Press, Cambridge, UK, 1995.

Huang, N. E. and Wu, Z.: A review on Hilbert-Huang transform: method and its applications to geophysical studies, Rev. Geophys., 46, 1-23, doi:10.1029/2007RG000228 2008.

Huang, N. E., Shen, Z., Long, S. R., Wu, M. C., Shih, H. H., Zheng, Q., Yen, N.-C., Tung, C. C., and Liu, H. H.: The empirical mode decomposition and the Hilbert spectrum for nonlinear and non-stationary time series analysis, P. Roy. Soc. Lond. A, 454, 903-995, 1998.

Huang, N. E., Shen, Z., and Long, S. R.: A new view of nonlinear water waves: the Hilbert Spectrum 1, Annu. Rev. Fluid Mech., 31, 417-457, 1999.

Huang, Y., Schmitt, F. G., Lu, Z., and Liu, Y.: An amplitudefrequency study of turbulent scaling intermittency using empirical mode decomposition and Hilbert spectral analysis, Europhys. Lett., 84, 40010, doi:10.1209/0295-5075/84/40010 2008.

Huang, Y., Schmitt, F. G., Hermand, J.-P., Gagne, Y., Lu, Z., and Liu, Y.: Arbitrary-order Hilbert spectral analysis for time series possessing scaling statistics: comparison study with detrended fluctuation analysis and wavelet leaders, Phys. Rev. E, 84, 016208, doi:10.1103/PhysRevE.84.016208, 2011.
Jackson, G. A., Maffione, R., Costello, D. K., Alldredge, A. L., Logan, B. E., and Dam, H. G.: Particle size spectra between $1 \mathrm{~mm}$ and $1 \mathrm{~cm}$ at Monterey Bay determined using multiple instruments, Deep-Sea Res. Pt. I, 44, 1739-1767, 1997.

Jiang, Q. and Logan, B. E.: Fractal dimensions of aggregates determined from steady-state size distributions, Environ. Sci. Technol., 25, 2031-2038, 1991.

Jonaszz, M.: Particle-size distributions in the Baltic, Tellus B, 35, 346-358, 1983.

Jouon, A., Ouillon, S., Douillet, P., Lefebvre, J. P., Fernandez, J. M., Mari, X., and Froidefond, J.-M.: Spatio-temporal variability in suspended particulate matter concentration and the role of aggregation on size distribution in a coral reef lagoon, Mar. Geol., 256, 36-48, 2008.

Kerman, B. R.: A multifractal equivalent of the Beaufort scale for sea-state, Geophys. Res. Lett., 20, 297-300, 1993.

Kitchen, J., Zaneveld, J. R. V., and Pak, H.: Effect of particle size distribution and chlorophyll content on beam attenuation spectra, Appl. Optics, 21, 3913-3918, 1982.

Lefebvre, J.-P., Ouillon, S., Vinh, V. D., Arfi, R., Panché, J.-Y., Mari, X., Van Thuoc, C., and Torréton, J.-P.: Seasonal variability of cohesive sediment aggregation in the Bach Dang-Cam Estuary, Haiphong (Vietnam), Geo-Mar. Lett., 32, 103-121, 2012.

Li, D. H. and Ganczarczyk, J.: Fractal geometry of particle aggregates generated in water and wastewater treatment processes, Environ. Sci. Technol., 23, 1385-1389, 1989.

Logan, B. E. and Wilkinson, D. B.: Fractal dimensions and porosities of Zoogloea ramigera and Saccharomyces cerevisae aggregates, Biotechnol. Bioeng., 38, 389-396, 1991.

Loisel, H., Nicolas, J.-M., Sciandra, A., Stramski, D., and Poteau, A.: Spectral dependency of optical backscattering by marine particles from satellite remote sensing of the global ocean, J. Geophys. Res., 111, C09024, doi:10.1029/2005JC003367 2006.

Lovejoy, S. and Schertzer, D.: Multifractals, cloud radiances and rain, J. Hydrol., 322, 59-88, 2006.

Lovejoy, S. and Schertzer, D.: Haar wavelets, fluctuations and structure functions: convenient choices for geophysics, Nonlin. Processes Geophys., 19, 513-527, doi:10.5194/npg-19-513-2012, 2012.

Lovejoy, S. and Schertzer, D.: The Weather and Climate: Emergent Laws and Multifractal Cascades, Cambridge University Press, Cambridge, UK, 2013.

Lovejoy, S., Currie, W., Tessier, Y., Claereboudt, M., Bourget, E., Roff, J., and Schertzer, D.: Universal multifractals and ocean patchiness: phytoplankton, physical fields and coastal heterogeneity, J. Plankton Res., 23, 117-141, 2001a.

Lovejoy, S., Schertzer, D., Tessier, Y., and Gaonac'h, H.: Multifractals and resolution-independent remote sensing algorithms: the example of ocean colour, Int. J. Remote Sens., 22, 1191-1234, $2001 \mathrm{~b}$.

McCave, I. N.: Particulate size spectra, behavior, and origin of nepheloid layers over the Nova Scotian continental rise, J. Geophys. Res., 88, 7647-7666, 1983.

Neukermans, G., Loisel, H., Mériaux, X., Astoreca, R., and McKee, D.: In situ variability of mass-specific beam attenuation and backscattering of marine particles with respect to particle size, density, and composition, Limnol. Oceanogr., 57, 124-144, 2012. 
Nieves, V., Llebot, C., Turiel, A., Solé, J., García-Ladona, E., Estrada, M., and Blasco, D.: Common turbulent signature in sea surface temperature and chlorophyll maps, Geophys. Res. Lett., 34, L23602, doi:10.1029/2007GL030823, 2007.

Pope, S. B.: Turbulent Flows, Cambridge University Press, Cambridge, UK, 2000.

Pottier, C., Turiel, A., and Garçon, V.: Inferring missing data in satellite chlorophyll maps using turbulent cascading, Remote Sens. Environ., 112, 4242-4260, 2008.

Renosh, P. R., Schmitt, F. G., Loisel, H., Sentchev, A., and Mériaux, X.: High frequency variability of particle size distribution and its dependency on turbulence over the sea bottom during resuspension processes, Cont. Shelf Res., 77, 51-60, 2014.

Renosh, P. R., Schmitt, F. G., and Loisel, H.: Scaling analysis of ocean surface turbulent heterogeneities from satellite remote sensing: use of 2D structure functions, PLoS ONE, 10, e0126975, doi:10.1371/journal.pone.0126975, 2015.

Reynolds, R., Stramski, D., Wright, V., and Woźniak, S.: Measurements and characterization of particle size distributions in coastal waters, J. Geophys. Res.-Oceans, 115, C08024, doi:10.1029/2009JC0059302010.

Schertzer, D., Lovejoy, S., Schmitt, F., Chigirinskaya, Y., and Marsan, D.: Multifractal cascade dynamics and turbulent intermittency, Fractals, 5, 427-471, 1997.

Schmitt, F. G. and Huang, Y.: Stochastic Analysis of Scaling Time Series: From Turbulence Theory to Applications, Cambridge University Press, Cambridge, UK, 2015.

Schmitt, F. G., Huang, Y., Lu, Z., Liu, Y., and Fernandez, N.: Analysis of velocity fluctuations and their intermittency properties in the surf zone using empirical mode decomposition, J. Mar. Syst., 77, 473-481, 2009.

Seuront, L., Schmitt, F., Lagadeuc, Y., Schertzer, D., Lovejoy, S., and Frontier, S.: Multifractal analysis of phytoplankton biomass and temperature in the ocean, Geophys. Res. Lett., 23, 35913594, 1996a.

Seuront, L., Schmitt, F., Schertzer, D., Lagadeuc, Y., and Lovejoy, S.: Multifractal intermittency of Eulerian and Lagrangian turbulence of ocean temperature and plankton fields, Nonlin. Processes Geophys., 3, 236-246, doi:10.5194/npg-3-236-1996, $1996 b$.
Seuront, L., Schmitt, F., Lagadeuc, Y., Schertzer, D., and Lovejoy, S.: Universal multifractal analysis as a tool to characterize multiscale intermittent patterns: example of phytoplankton distribution in turbulent coastal waters, J. Plankton Res., 21, 877-922, 1999.

Sheldon, R., Prakash, A., and Sutcliffe, W.: The size distribution of particles in the ocean, Limnol. Oceanogr., 17, 327-340, 1972.

Squires, K. D. and Eaton, J. K.: Preferential concentration of particles by turbulence, Phys. Fluids A, 3, 1169-1178, 1991.

Stramski, D. and Kiefer, D. A.: The size distribution of particles in the ocean, Prog. Oceanogr., 28, 343-383, 1991.

Svendsen, I. A.: Analysis of surf zone turbulence, J. Geophys. Res.Oceans, 92, 5115-5124, 1987.

Tessier, Y., Lovejoy, S., and Schertzer, D.: Universal multifractals: theory and observations for rain and clouds, J. Appl. Meteorol., 32, 223-250, 1993.

Traykovski, P., Latter, R. J., and Irish, J. D.: A laboratory evaluation of the laser in situ scattering and transmissometery instrument using natural sediments, Mar. Geol., 159, 355-367, 1999.

Turiel, A., Nieves, V., Garcia-Ladona, E., Font, J., Rio, M.-H., and Larnicol, G.: The multifractal structure of satellite sea surface temperature maps can be used to obtain global maps of streamlines, Ocean Sci., 5, 447-460, doi:10.5194/os-5-447-2009, 2009.

Twardowski, M. S., Boss, E., Macdonald, J. B., Pegau, W. S., Barnard, A. H., and Zaneveld, J. R. V.: A model for estimating bulk refractive index from the optical backscattering ratio and the implications for understanding particle composition in case I and case II waters, J. Geophys. Res.-Oceans, 106, 14129-14142, 2001.

Vulpiani, A. and Livi, R.: The Kolmogorov Legacy in Physics, Springer Science and Business Media, Berlin, 2003.

Wu, M. K. and Friedlander, S. K.: Note on the power law equation for fractal-like aerosol agglomerates, J. Colloid Interf. Sci., 159, 246-248, 1993.

Yoshimoto, H. and Goto, S.: Self-similar clustering of inertial particles in homogeneous turbulence, J. Fluid Mech., 577, 275-286, 2007. 